\title{
The Potential for Greater Clarity Cryo-Electron Microscopy via Ptychography
}

Timothy J. Pennycook ${ }^{1,2}$, Gerardo T. Martinez ${ }^{3}$, Peter D. Nellist ${ }^{3}$ and Jannik C. Meyer ${ }^{2}$

1. Stuttgart Center for Electron Microscopy, Max Planck Institute for Solid State Research, Stuttgart, Germany.

2. Faculty of Physics, University of Vienna, Vienna, Austria.

3. Department of Materials, University of Oxford, Oxford, UK.

For many applications in both the biological and materials sciences, finite dose tolerance and noise severely restrict the resolving power of microscopy. Without sufficient signal to overcome noise, microscopy cannot provide images at the full resolution of the optics. This has led to the rise of techniques such as cryo-electron microscopy (cryo-EM) and single particle analysis. By freezing samples, cryo-EM enhances their robustness to the electron beam. However many specimens remain extremely fragile in cryo-EM, and structure determination is only possible via single particle analysis, in which large numbers of particles with the same structure are imaged. Images of the structure in the same orientation are identified and averaged to enhance the signal to noise. From these images models are built of the 3D structure of the particles and optimized to match the experimental data. The technique has been considered revolutionary, as it has enabled high resolution imaging of molecules that resist the crystallization that is required for X-ray crystallography [1]. However the quality of the reconstruction still depends on the clarity of the raw images. For instance, the quality of the alignment of the images of the individual particles will increase as they themselves increase in clarity. Thus methods that provide enhanced clarity at low doses will help to push the limits of cryo-EM, and provide enhanced resolution.

Cryo-EM and single particle analysis have benefited greatly from recent developments in pixelated detector technology. The much enhanced detection quantum efficiency (DQE) of direct electron detectors has translated directly into greater signal to noise in the raw images of the individual particles, in turn greatly enhancing the resolution of the single particle reconstructions. Phase contrast imaging in the high-resolution transmission electron microscope (HRTEM) has remained the tool of choice for cryo-EM single particle analysis due to its high dose efficiency. However advances in pixelated detector technology are also enhancing the ability of scanning transmission electron microscopy (STEM) to perform efficient phase contrast imaging via electron ptychography [2].

Here we will highlight the potential for pushing the limits of cryo-EM by using ptychographic phase contrast in the STEM in place of HRTEM imaging. Ptychography provides greater clarity phase contrast than HRTEM via a combination of double resolution, and strong robustness to partial coherence [3]. This clarity is illustrated in Figure 1 with simulated HRTEM and ptychographic STEM images of an andenosine triphosphate (ATP) molecule at a dose of $20,000 \mathrm{e}^{-} / \AA^{2}$ and an accelerating voltage of $80 \mathrm{kV}$. For the HRTEM simulations the defocus was set to $2 \mathrm{~nm}$ and Cs to zero as these parameters were found to maximize the contrast. No such injection of aberrations is needed for ptychography, and the ptychographic image was simulated assuming an aberration free probe using the single-side band method [2]. The ptychographic image includes partial temporal coherence with a realistic $3.2 \mathrm{~nm}$ defocus spread and is far clearer than the equivalent partially coherent HRTEM image with the same defocus spread. Even the perfectly coherent HRTEM image appears less sharp than the partially coherent ptychographic image because of the double resolution of ptychography. All three images used a $35 \mathrm{mrad}$ aperture, but whereas HRTEM passes frequencies up to the aperture cutoff, ptychography 
passes frequencies up to twice the aperture cutoff. By performing cryo-EM and single particle analysis with ptychography in STEM it should be possible to realize this gain in clarity experimentally for ultra low dose imaging. The same advantages shown here at $20,000 \mathrm{e}^{-/} \AA^{2}$ apply equally to ptychography performed at doses below $50 \mathrm{e}^{-} / \AA^{2}$.

By performing phase imaging in STEM without the need for aberrations to form contrast, one gains the additional advantage of the ability to simultaneously acquire other more compositionally sensitive signals. At very low doses individual annular dark field (ADF) and energy-dispersive X-ray spectroscopy (EDX) images will be too noisy to display much if any structure, but by aligning enough frames based on the phase images, sufficient signal to noise can be built up to also provide atomic resolution $\mathrm{ADF}$ and spectrum images at ultra low doses in single particle analysis. This is a powerful combination of signals. While structure is most clearly resolved by phase imaging at low doses, ADF and spectroscopy provide far greater direct sensitivity to composition. Ptychographic phase contrast imaging can also be corrected for aberrations after acquiring the data using a Wigner distribution deconvolution (WDD) approach [4] as illustrated with simulations in Figure 2, meaning one can avoid spending time and dose fine tuning the conditions, a great advantage for beam sensitive materials. It also presents the possibility of performing optical sectioning with just a single scan [4], potentially providing very large dose savings for imaging in 3D.

\section{References:}

[1] E. Callaway, Nature 525, (2015) p. 172.

[2] T. J. Pennycook et al., Ultramicrosopy 151 (2015), p. 160.

[3] T. J. Pennycook et al., Submitted for publication.

[4] H. Yang et al., Nat. Comms. 7 (2016), p. 12532.

[5] Research funded by the EU Marie Skłodowska-Curie grant No. 655760 - DIGIPHASE (TJP).
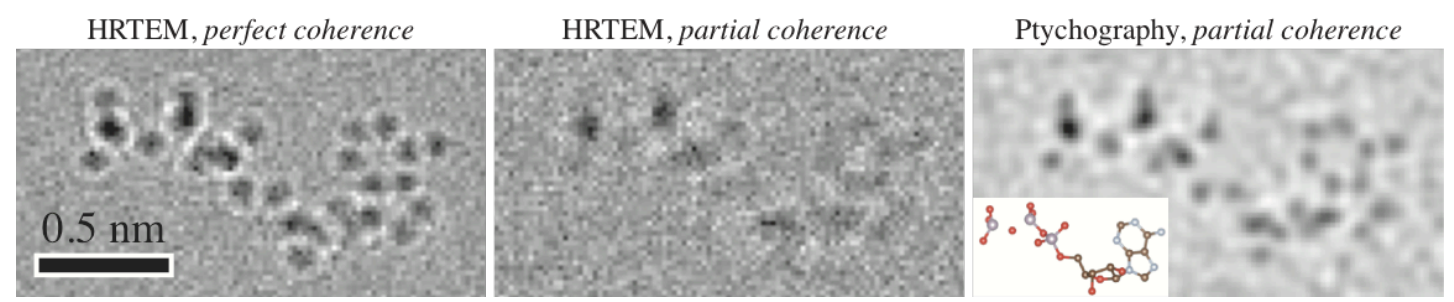

Figure 1. HRTEM and ptychographic phase imaging of an ATP molecule simulated at 20,000 $\mathrm{e}^{-} / \AA^{2}$.

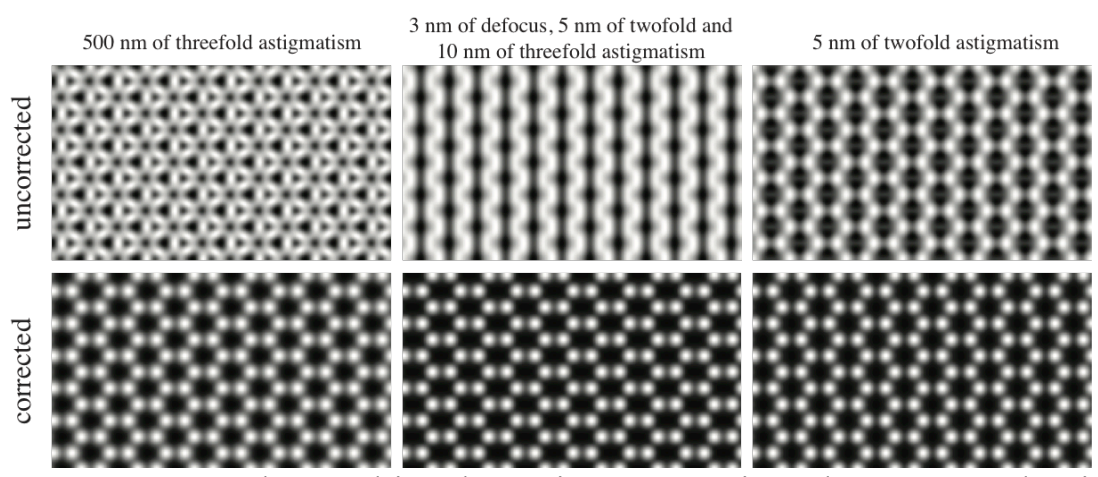

Figure 2. Ptychographic aberration correction demonstrated with data of graphene simulated with known amounts of aberrations that the correction algorithm is blind to. 